\title{
X-RAY STUDIES OF THE BLACK WIDOW PULSAR PSR B1957+20
}

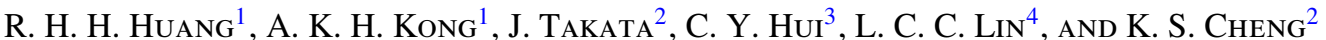 \\ ${ }^{1}$ Institute of Astronomy and Department of Physics, National Tsing Hua University, Hsinchu, Taiwan \\ ${ }^{2}$ Department of Physics, University of Hong Kong, Pokfulam Road, Hong Kong \\ ${ }^{3}$ Department of Astronomy and Space Science, Chungnam National University, Daejeon, Republic of Korea \\ ${ }^{4}$ General Education Center of China Medical University, Taichung, Taiwan \\ Received 2012 March 13; accepted 2012 September 25; published 2012 November 6
}

\begin{abstract}
We report on Chandra observations of the black widow pulsar, PSR B1957+20. Evidence for a binary-phase dependence of the X-ray emission from the pulsar is found with a deep observation. The binary-phase-resolved spectral analysis reveals non-thermal X-ray emission of PSR B1957+20, confirming the results of previous studies. This suggests that the X-rays are mostly due to intra-binary shock emission, which is strongest when the pulsar wind interacts with the ablated material from the companion star. The geometry of the peak emission is determined in our study. The marginal softening of the spectrum of the non-thermal X-ray tail may indicate that particles injected at the termination shock are dominated by synchrotron cooling.
\end{abstract}

Key words: binaries: eclipsing - stars: individual (PSR B1957+20) - stars: neutron

Online-only material: color figures

\section{INTRODUCTION}

The widely accepted scenario for the formation of a millisecond pulsar (MSP) is that an old neutron star has been spun up to millisecond periods in a past accretion phase by mass and angular momentum transfer from a binary late-type companion (Alpar et al. 1982; Radhakrishnan \& Srinivasan 1982). Once the accretion has stopped, the relativistic magnetized pulsar wind, which is believed to carry away the pulsar rotational energy and angular momentum, may be able to ablate and eventually evaporate its companion (Rasio et al. 1989). Close binary systems with MSPs are a subject of special interest, since they are thought to be the missing link between low-mass X-ray binaries and isolated MSPs. Discoveries of eclipsing binary pulsar systems, such as PSR B1957+20 (Fruchter et al. 1988b), PSR J2051-0827 (Stappers et al. 1996), and PSR J1023+0038 (Archibald et al. 2009, 2010) in the Galactic plane and 47 Tuc W (PSR J0024-7204W; Bogdanov et al. 2005) and PSR J1740-5340 (D'Amico et al. 2001) in globular clusters, gave support to this formation scenario. Studying these binary systems provides a wealth of information, not only on the evolutionary history of isolated MSPs, but also on the physical details of the pulsar's high-energy emission properties.

PSR B1957+20 was discovered at Arecibo in 1988 (Fruchter et al. 1988b). It is in a binary system with a $0.025 M_{\odot}$ companion in a $9.16 \mathrm{hr}$ orbital period. The pulsar has a spin period of $1.6 \mathrm{~ms}$, the third shortest among all known MSPs. Its period derivative of $\dot{P}=7.85 \times 10^{-21} \mathrm{~s} \mathrm{~s}^{-1}$ implies a spin-down energy of $\dot{E}=$ $7.48 \pm 3.61 \times 10^{34} \mathrm{erg} \mathrm{s}^{-1}$, a characteristic spin-down age of $>$ $3.4 \times 10^{9} \mathrm{yr}$, and a dipole surface magnetic field of $B_{\perp}=1.12 \times$ $10^{8} \mathrm{G}$ (Guillemot et al. 2012). For a radio dispersion measure inferred distance of $2.5 \pm 1.0 \mathrm{kpc}$ (Cordes \& Lazio 2002), the pulsar moves through the sky with a supersonic velocity of $220 \mathrm{~km} \mathrm{~s}^{-1}$ (Arzoumanian et al. 1994). The interaction of a relativistic wind flowing away from the pulsar with the interstellar medium (ISM) produces an $\mathrm{H} \alpha$ bow shock nebula which was the first nebula to be found around a "recycled" pulsar (Kulkarni \& Hester 1988). For approximately $10 \%$ of this orbit, the radio emission at a frequency of $430 \mathrm{MHz}$ from the pulsar is eclipsed by material ablated by the pulsar wind from the companion (Fruchter et al. 1988b). Optical observations by Fruchter et al. (1988a) and van Paradijs et al. (1988) revealed that the pulsar wind consisting of electromagnetic radiation and high-energy particles is ablating and evaporating its white dwarf companion star. This rarely observed property gave the pulsar the name black widow pulsar. Subsequent analyses of the optical light curve for the binary system by Reynolds et al. (2007) placed a constraint on the system inclination of $65^{\circ} \pm 2^{\circ}$ for a pulsar in the mass range $1.3-1.9 M_{\odot}$. The effective temperatures of $T=2900 \pm 110 \mathrm{~K}$ and $T=8300 \pm 200 \mathrm{~K}$ for the unilluminated side and the illuminated side of the companion were also obtained in their studies.

Several X-ray studies of PSR B1957+20 have been carried out in the past two decades (Kulkarni et al. 1992; Stappers et al. 2003; Huang \& Becker 2007). The X-ray emission of PSR B1957+20 is found to be non-thermal dominated and best modeled with a single power-law (PL) spectrum, which indicates that the $\mathrm{X}$-rays originate from the shock interaction of the pulsar wind with the wind of the companion star or from the pulsar magnetosphere (Stappers et al. 2003; Huang \& Becker 2007). Searching for an X-ray pulsation at the radio pulsar's rotation period and a modulation of the X-ray emission from the PSR B1957+20 system with orbital phase is another important subject, which may help to discriminate the origin of the X-ray emission. Recently, a $4 \sigma$ detection of X-ray coherent pulsation was reported by Guillemot et al. (2012). In addition, Huang \& Becker (2007) found a strong correlation of the pulsar's X-ray flux with its orbital period. However, due to the short exposure, we could not determine whether the flux modulation was periodic, and given the limited photon statistics it was not possible to investigate any spectral variation as a function of orbit phase or to determine the exact geometry of the peak emission. Repeated coverage of the binary orbit in a longer Chandra observation would provide us with a better photon statistic and allow us to determine the emission geometry with higher accuracy.

In this paper, we report on an archival Chandra observation of the black widow pulsar. This investigation offers further insight 

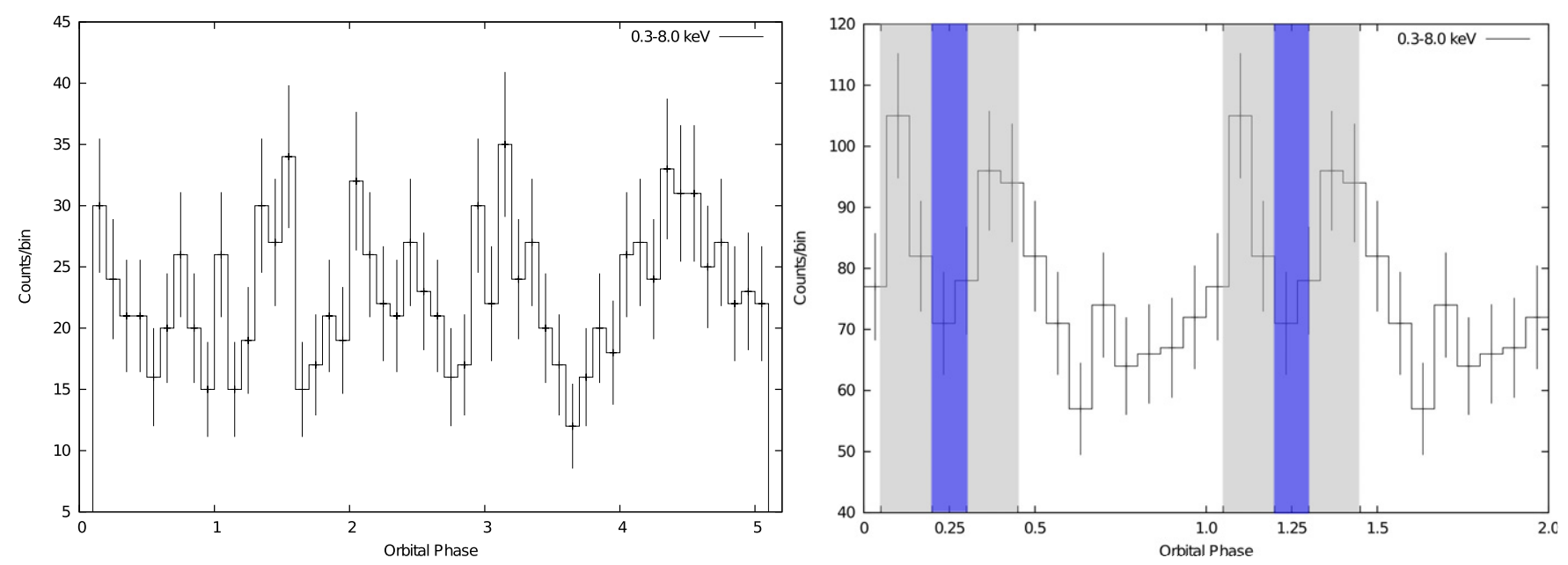

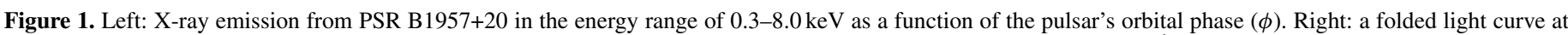

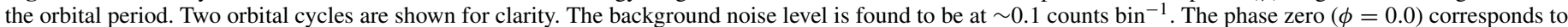

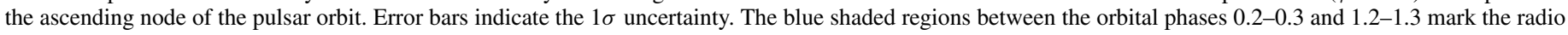
eclipse of the black widow pulsar. The phase-resolved spectrum covering the eclipsing region was extracted from the gray shaded regions (see Section 5.1).

(A color version of this figure is available in the online journal.)

into the properties of this remarkable binary. In Section 2, we summarize the details of the Chandra observation and the data reduction. A search for the X-ray orbital modulation from this system is described in Section 3. In Sections 4 and 5, we present the results of the X-ray spatial and spectral analysis. Finally, we discuss the physical implications of the observed results in Section 6.

\section{OBSERVATION}

A Chandra observation aimed at PSR B1957+20 was performed on 2008 August 15 (ObsID 9088) using the backilluminated chip ACIS-S3 with an uninterrupted 169 ks exposure. The data were configured in the VFAINT telemetry mode. Data reduction and analysis were processed with Chandra Interactive Analysis Observations (CIAO) version 4.3 software and the Chandra Calibration Database (CALDB) version 4.4.1. The level 1 data with background cleaning were used in our study. Data analysis was restricted to the energy range of $0.3-8.0 \mathrm{keV}$.

For the timing and spectral analyses of the black widow pulsar, we extracted the photons from a circular region centered at the radio timing position, ${ }^{5}$ R.A. $(\mathrm{J} 2000)=19^{\mathrm{h}} 59^{\mathrm{m}} 36^{\mathrm{s}} .77$, decl. $=20^{\circ} 48^{\prime} 15^{\prime \prime} .12$, with a radius of $2^{\prime \prime}$ which encloses $90 \%$ of the total source energy at $1.5 \mathrm{keV}$.

\section{TIMING ANALYSIS}

For the timing analysis, we first extracted the photons from the aforementioned circle and translated the photon arrival times to the solar system barycenter by using the CIAO tool axbary. The JPL DE200 solar system ephemeris was used for the barycentric correction to ensure consistency with the radio ephemeris. We note that searching for the X-ray pulses at the spin period of PSR B1957+20 was precluded by the inappropriate temporal resolution of this Chandra observation with a frame time of $3.2 \mathrm{~s}$.

As the Chandra observation covers over five consecutive binary orbits, by plotting a light curve of the X-ray source counts versus the orbital phase (see Figure 1, left panel), we

\footnotetext{
5 From the ATNF Pulsar Catalogue.
}

can confirm that the X-ray flux is not steady with time. We also applied a Kolmogorov-Smirnov (K-S) test to the unbinned light curve data in order to have a bin-independent statistical evaluation of the X-ray emission variability. Calculating the corresponding $\mathrm{K}-\mathrm{S}$ probabilities between our data set and the cumulative distribution function, generated by assuming a constant X-ray flux, indicated a marginal deviation between these two distributions. The significance for an intra-orbital flux modulation from this test is only $\sim 81 \%$. A more significant result, i.e., $\sim 96 \%$, is obtained if we restrict the analysis to the soft energy band below $2 \mathrm{keV}$.

In order to search for a modulation of the X-ray flux as a function of the orbital phase, we first selected X-ray data covering five complete and consecutive orbits and then used the radio timing ephemeris of PSR B 1957+20 from a pulsar catalog provided by Lucas Guillemot ${ }^{6}$ to fold a light curve at the orbital period (see Figure 1, right panel). Using a $\chi^{2}$-test, the significance for a flux modulation over the observed orbit was found to be $\sim 99 \%$.

\section{SPATIAL ANALYSIS}

Figure 2 (left panel) shows the Chandra ACIS-S3 image in the energy band $0.3-8 \mathrm{keV}$ of the field around PSR B1957+20. This image was created by using an adaptive smoothing algorithm with a Gaussian kernel of $\sigma<3$ pixels in order to probe the detailed structure of faint diffuse emission. Both the pulsar and an extended X-ray feature (hereafter the "tail"), protruding from the pulsar position, can be clearly seen in this image. The length of the tail, with its orientation to the northeast, is about 25 arcsec. The right panel in Figure 2 presents the $\mathrm{H} \alpha$ image overlaid with the X-ray contours. This $\mathrm{H} \alpha$ image is obtained from Taurus Tunable Filter service mode observations on the Anglo Australian Telescope in 2000. An $\mathrm{H} \alpha$ bow shock emission aligned with the proper-motion direction of the PSR B1957+20 system fades to the background in $\sim 40^{\prime \prime}$. Compared with the previous result reported by Stappers et al. (2003), the X-ray tail is more extended due to the longer exposure time of this Chandra observation.

\footnotetext{
6 ftp://www.cenbg.in2p3.fr/astropart/lucas/report/1959+2048.html
} 


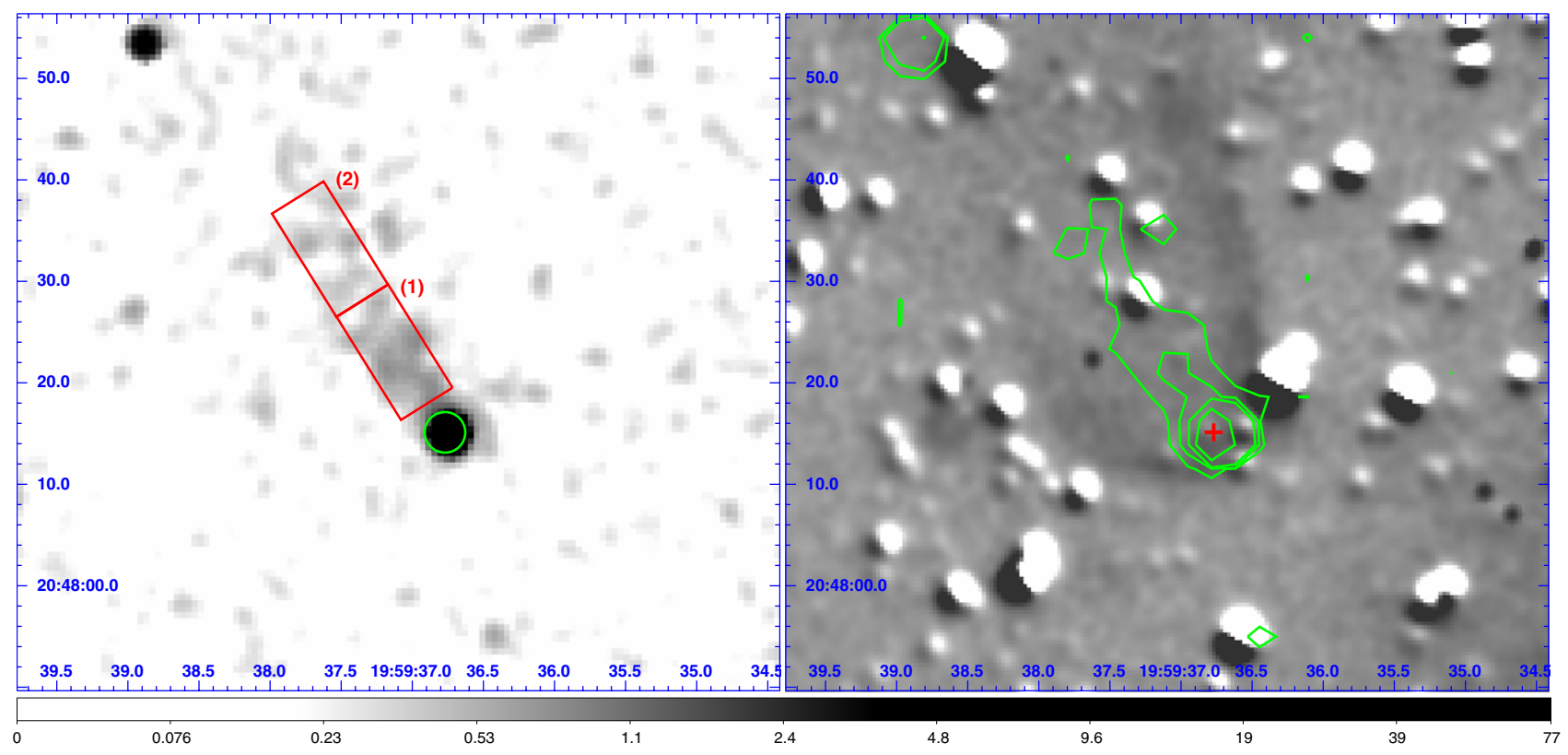

Figure 2. Left: Chandra ACIS-S3 image in the energy band $0.3-8 \mathrm{keV}$ of the black widow pulsar system smoothed with an adaptive Gaussian filter. The green circle with the 2 .". 0 radius indicates the source region we used in this study while two segments of the X-ray tail are chosen from the red rectangular regions. Right: the H $\alpha$ image taken from the Anglo Australian Telescope is overlaid with the X-ray contours. The green contour levels are shown at $0.4 \%, 0.8 \%, 3.0 \%, 15.4 \%$, and $84.8 \%$ of the peak X-ray surface brightness. The red cross indicates the radio timing position of PSR B1957+20. The optical residuals correspond to incompletely subtracted stars.

(A color version of this figure is available in the online journal.)

\section{SPECTRAL ANALYSIS}

\subsection{PSR B1957+20}

We used the CIAO tool dmextract to extract spectra of the source and two nearby source-free background regions. Response files were constructed by using the CIAO tools mkacisrmf and mkarf. The extracted spectra were binned with at least 30 source counts bin ${ }^{-1}$. Background-subtracted spectral modeling was performed with XSPEC (version 12.7.0) using data in the energy band $0.3-8.0 \mathrm{keV}$.

Assuming that the X-ray emission originates from the intrabinary shock or the pulsar's magnetosphere (Stappers et al. 2003; Huang \& Becker 2007; Guillemot et al. 2012), we expect the radiation to be synchrotron. To test this hypothesis, we fitted the spectrum with an absorbed PL model. Unexpectedly, a single PL model cannot provide any statistically acceptable description of the observed spectrum (i.e., $\chi_{v}^{2}>2$ ). We then tested whether a single blackbody (BB) model, a double PL model, or a composite spectral model consisting of a PL and a BB can provide an appropriate modeling of the data. Neither the one-component BB model nor the doublecomponent PL model yields any physically acceptable fits. Instead, the composite model gives a better description of the $\mathrm{X}$-ray spectrum. The inferred temperatures of $0.18 \mathrm{keV}$ and $0.30 \mathrm{keV}$ are much too high for the cooling surface of the neutron star, but may be explained by the heated polar cap region (cf. Zavlin 2007; Takata et al. 2012). Considering that a portion of the $\mathrm{X}$-ray emission could originate from a thermal plasma within or around the binary system, possibly from the active corona of the secondary star or the plasma responsible for the radio eclipses (Bogdanov et al. 2011), a MEKAL, a thermal bremsstrahlung (TB), a MEKAL+PL, and a PL+TB model were tested in our study. We found that those models cannot provide a better or an acceptable description of the $\mathrm{X}$-ray spectrum of the pulsar system. Besides, we also examined the X-ray spectrum with more complicated spectral models, e.g., PL+PL+BB or PL+BB+BB. Although an acceptable fit can be yielded by a three-component model, i.e., PL+BB+BB, no physical interpretation can be appropriately applied to this binary system. Therefore, we suspect a dependence of the X-ray spectrum of PSR B1957+20 on its orbital phase due to the variability observed in its X-ray flux level which seems to correlate with its orbital period.

To investigate whether the X-ray spectral behavior of PSR B1957+20 varies across the orbit, we analyzed the X-ray spectra within the orbital phase of $\phi=0.05-0.45$, which covers the ingress, eclipsing, and egress region and outside the aforementioned region $(\phi=0.45-1.05)$, separately. The source and background spectra were extracted from the same circles as mentioned before and dynamically binned in accordance with the photon statistic in each data set. The net count rates for the spectral analysis inside and outside the eclipsing region are $7.49 \pm 0.33$ and $5.88 \pm 0.25$ counts ks ${ }^{-1}$, respectively. We found that the binary-phase-resolved spectral analysis reveals a nonthermal emission nature of the detected X-rays and each of the observed spectra can be well described by a single PL model with different photon indices, which indicates that its spectral behavior is orbital dependent.

The spectral parameters inferred from the PL fits as well as the best-fit hydrogen column density $N_{\mathrm{H}}$ are found to be consistent with those reported by Stappers et al. (2003) and Huang \& Becker (2007). For comparison, a fixed $N_{\mathrm{H}}$ value of $1.8 \times 10^{21}$ $\mathrm{cm}^{-2}$ obtained by Stappers et al. (2003) is also applied in our spectral analysis. The results of the spectral fits are summarized in Table 1.

\subsection{The X-Ray Tail}

Stappers et al. (2003) uncovered an X-ray tail with a position angle opposite to the proper-motion direction of PSR B 1957+20 with a Chandra observation taken in 2001. However, with low photon statistics, a detailed spectral analysis was restricted. The 
Table 1

X-Ray Spectral Parameters of the PSR B1957+20 Binary System

\begin{tabular}{|c|c|c|c|c|c|}
\hline Orbital Phase & Model & $\begin{array}{c}N_{\mathrm{H}} \\
\left(10^{21} \mathrm{~cm}^{-2}\right)\end{array}$ & $\begin{array}{l}\Gamma / k T \\
(\mathrm{keV})\end{array}$ & $\begin{array}{c}F_{\mathrm{X}}^{\mathrm{a}} \\
\left(10^{-14} \mathrm{erg} \mathrm{cm}^{-2} \mathrm{~s}^{-1}\right)\end{array}$ & $\chi_{v}^{2} /$ dof \\
\hline \multicolumn{6}{|c|}{ Pulsar } \\
\hline \multirow[t]{4}{*}{$0.0-1.0$} & PL & $0.83_{-0.32}^{+0.33}$ & $1.96 \pm 0.12$ & $6.05_{-0.28}^{+0.26}$ & $1.86 / 18$ \\
\hline & PL & $1.80^{\mathrm{b}}$ & $2.26^{\mathrm{c}}$ & $7.22_{-0.14}^{+0.13}$ & $2.17 / 19$ \\
\hline & $\mathrm{PL}+\mathrm{BB}$ & $<0.61$ & $\Gamma=1.02_{-0.31}^{+0.37}, k T=0.30_{-0.05}^{+0.02}$ & $5.39_{-1.14}^{+0.06}$ & $1.48 / 16$ \\
\hline & $\mathrm{PL}+\mathrm{BB}$ & $1.80^{\mathrm{b}}$ & $\Gamma=1.66_{-0.25}^{+0.22}, k T=0.18_{-0.03}^{+0.02}$ & $7.18_{-0.28}^{+0.12}$ & $1.75 / 17$ \\
\hline \multirow[t]{2}{*}{$0.05-0.45$} & PL & $0.78_{-0.53}^{+0.56}$ & $1.80_{-0.17}^{+0.19}$ & $8.05_{-0.70}^{+0.53}$ & $0.75 / 13$ \\
\hline & PL & $1.80^{\mathrm{b}}$ & $2.09 \pm 0.10$ & $9.23_{-0.39}^{+0.43}$ & $0.92 / 14$ \\
\hline \multirow[t]{2}{*}{$0.45-0.05$} & PL & $0.89_{-0.50}^{+0.54}$ & $2.17_{-0.20}^{+0.21}$ & $6.12_{-0.38}^{+0.36}$ & $0.99 / 15$ \\
\hline & PL & $1.80^{\mathrm{b}}$ & $2.49_{-0.10}^{+0.11}$ & $7.61_{-0.19}^{+0.24}$ & $1.10 / 16$ \\
\hline Segment & Model & $\begin{array}{c}N_{\mathrm{H}} \\
\left(10^{21} \mathrm{~cm}^{-2}\right)\end{array}$ & $\Gamma$ & $\begin{array}{c}F_{\mathrm{x}}^{\mathrm{a}} \\
\left(10^{-14} \mathrm{erg} \mathrm{cm}^{-2} \mathrm{~s}^{-1}\right)\end{array}$ & $\chi_{v}^{2} / \mathrm{dof}$ \\
\hline \multicolumn{6}{|c|}{ Tail } \\
\hline \multirow[t]{2}{*}{ Whole tail } & PL & $2.77_{-2.42}^{+3.21}$ & $2.62_{-0.84}^{+1.23}$ & $0.89_{-0.19}^{+0.10}$ & $0.77 / 4$ \\
\hline & PL & $1.80^{\mathrm{b}}$ & $2.30_{-0.36}^{+0.39}$ & $0.71_{-0.07}^{+0.10}$ & $0.64 / 5$ \\
\hline Region 1 & PL & \multirow{2}{*}{$1.80^{\mathrm{b}}$} & $1.57_{-0.26}^{+0.31}$ & $0.46_{-0.10}^{+0.13}$ & \multirow{2}{*}{$1.28 / 18$} \\
\hline Region 2 & PL & & $2.14_{-0.37}^{+0.46}$ & $0.33_{-0.05}^{+0.16}$ & \\
\hline
\end{tabular}

Notes.

${ }^{\text {a }}$ Unabsorbed X-ray flux in the energy range of $0.3-8.0 \mathrm{keV}$.

b The hydrogen column density $N_{\mathrm{H}}$ is fixed at $1.8 \times 10^{21} \mathrm{~cm}^{-2}$.

${ }^{\mathrm{c}}$ No error was calculated since the reduced $\chi^{2}$ is larger than the maximum value of two.

much longer exposure time of the latest archival Chandra observations allows us to study the $\mathrm{X}$-ray tail in greater detail. For comparison, we first selected a box of $16^{\prime \prime} \times 6^{\prime \prime}$ with an orientation along the proper-motion direction to be the region of the X-ray tail of PSR B1957+20. The background-subtracted count of $108 \pm 13$ was collected for the spectral analysis. With higher photon statistics, we found that an absorbed single PL model fits the X-ray spectrum of the tail well, which implies that the $\mathrm{X}$-ray emission originates from the pulsar's interaction with the ambient medium. Its non-thermal X-ray spectrum points to synchrotron emission from energetic particles from the pulsar wind.

A softening of the spectrum of the X-ray tail as a function of the distance from the pulsar is expected if synchrotron cooling of the particles injected at the termination shock is dominated. For the purpose of investigating the possible spectral variation, we performed a spatially resolved spectral analysis using two separate extraction regions along the tail. In the following, we refer to the segment close to the pulsar as region 1 and the further one as region 2 . The background-subtracted counts are $68 \pm 14$ for region 1 and $29 \pm 12$ for region 2, respectively. In order to better constrain the spectral properties, we followed the method adopted by Johnson \& Wang (2010) and Hui et al. (2012) to jointly fit individual PL models for the X-ray spectra of these two regions assuming that the column density, $N_{\mathrm{H}}$, does not change significantly along the proper-motion direction. Due to low photon statistics in each segment, we then fixed the $N_{\mathrm{H}}$ value at $1.8 \times 10^{21} \mathrm{~cm}^{-2}$ (Stappers et al. 2003) in the joint fit. The best-fit spectral parameters are shown in Table 1. An indication of such a spectral variation was found in this study.

\section{SUMMARY AND CONCLUSION}

We have searched for the orbital modulation of the X-ray emission from PSR B1957+20. Analyzing this data set with a $\chi^{2}$-test and a K-S test revealed a marginal intra-orbital flux modulation, which suggests that the non-thermal X-rays from PSR B1957+20 are mostly due to intra-shock emission at the interface between the pulsar wind and the ablated material from the companion star. The pulsar wind electrons and positrons are accelerated and randomized by the shock and emit the $\mathrm{X}$-rays via the synchrotron process. Such shock emission has also been suggested to explain the variable $\mathrm{X}$-ray flux as a function of orbital phase in 47 Tuc W (Bogdanov et al. 2005) and PSR J1740-5340 in NGC 6397 (Huang \& Becker 2010) and PSR J1023+0038 (Archibald et al. 2009, 2010; Tam et al. 2010; Bogdanov et al. 2011).

In Figure 1, the observed flux peaks just before and after the pulsar eclipse can be interpreted as the Doppler effect caused by the bulk flow in the downstream region. If the post-shocked wind flows toward (or away from) the Earth, then the Doppler effect increases (or decreases) the observed flux from the flux for an isotropic case. The shock geometry is controlled by the ratio of the momentum fluxes of the pulsar wind to the stellar wind (see Canto et al. 1996; Antokhin et al. 2004). For PSR B1957+20, the observed orbital period derivative $\dot{P}_{b} \sim 10^{-11}$ (Fruchter et al. 1990) suggests the mass-loss rate $\dot{M}_{*} \sim M_{*} \dot{P}_{b} P_{b}^{-1} \sim$ $10^{-10} M_{\odot} \mathrm{yr}^{-1}$, where $M_{*}=0.02 M_{\odot}$ and $P_{b}=33,001 \mathrm{~s}$ is the orbital period. With the mass-loss rate of $\dot{M} \sim 10^{-10} M_{\odot} \mathrm{yr}^{-1}$, the ratio of the momentum fluxes of the pulsar wind and the stellar wind is of the order of $\eta \equiv L_{\mathrm{sd}} / \dot{M}_{*} v_{\mathrm{es}} c \sim$ $10\left(L_{\mathrm{sd}} / 10^{35} \mathrm{erg} \mathrm{s}^{-1}\right)\left(\dot{M}_{*} / 10^{-10} M_{\odot} \mathrm{yr}^{-1}\right)\left(v_{\mathrm{es}} / 300 \mathrm{~km} \mathrm{~s}^{-1}\right)$, indicating that the companion star is confined by the pulsar wind and the shock. With momentum ratio $\eta \sim 10$, the opening angle of the cone-like shock is $\sim 50^{\circ}-60^{\circ}$ (see Eichler \& Usov 1993; Canto et al. 1996), which corresponds to $\sim 0.15$ orbital phase. Because the emission is concentrated in the forward direction of the flow, therefore, we expect that double peaks due to the 
Doppler effect appear at $\sim 0.15$ phase before and after the phase of radio eclipse, which is consistent with the observation.

As we can see in Figure 1, the observed ratio of maximum to minimum fluxes is two to three. For the emissions from the pulsar wind, the outgoing flux is modified by the Doppler effect as $F_{\nu}(E)=\mathcal{D}^{3} F_{v^{\prime}}^{\prime}\left(E^{\prime}\right)$ and $E=\mathcal{D} E^{\prime}$, with primed quantities refereeing to the comoving frame. Here, the relativistic boosting factor is given by

$$
\mathcal{D}=\frac{1}{\Gamma_{f}\left(1-\beta_{f} \cos \theta_{f}\right)},
$$

where $\Gamma_{f}$ and $\beta_{f}$ are the Lorentz factor and velocity in units of the speed of light of the flow, respectively, and $\theta_{f}$ is the angle between the Earth viewing angle and the direction of the flow. The ratio of maximum to minimum fluxes is estimated by

$$
\frac{F_{v, \text { max }}}{F_{v, \text { min }}} \sim\left(\frac{1-\beta_{f} \cos \theta_{f, \text { min }}}{1-\beta_{f} \cos \theta_{f, \text { max }}}\right)^{2+\alpha},
$$

where $\alpha$ is the photon index, and $\theta_{f, \max }$ and $\theta_{f, \text { min }} \sim \pi-\theta_{f, \text { max }}$ are typical angles at the orbital phases where the observed fluxes are maximum and minimum, respectively. For example, the observed ratio $F_{\max } / F_{\min } \sim 2-3$ and the photon index $\alpha \sim 2$ imply the flow velocity $\beta_{f} \sim 0.12-0.19$ for $\theta_{f, \max }=45^{\circ}$ and $\beta_{s} \sim 0.2-0.32$ for $\theta_{f, \max }=65^{\circ}$. Note that Guillemot et al. (2012) estimate the fraction of the pulsed emissions as about $30 \%$ of the total emissions. In such a case, the pulsed emissions may considerably contribute to the observed emissions at the off-peak orbital phase, and the intrinsic ratio of the maximum to minimum fluxes of the X-ray emissions from the inter-binary shock is larger than $F_{\max } / F_{\min } \sim 2-3$. This results in an increase in the flow velocity estimated from Equation (2).

Although the pulsed X-ray emission from PSR B1957+20 has been reported by Guillemot et al. (2012), the origin of the pulsed emissions is not known. As Table 1 shows, we find that the spectrum average over the whole orbit can be fitted better by a PL plus BB model. The effective temperature and radius of the $\mathrm{BB}$ component are $T_{\text {eff }} \sim 2 \times 10^{6} \mathrm{~K}$ and $R_{\text {eff }} \sim 0.1 \mathrm{~km}$, respectively, which are explained by the core component of the heated polar cap region (see Zavlin 2007; Takata et al. 2012). The observed flux of the BB component is $F_{\mathrm{BB}} \sim 2 \times 10^{-14} \mathrm{erg} \mathrm{cm}^{-2} \mathrm{~s}^{-1}$, which is several tens of a percent of the total emissions. This flux level of the pulsed emission is consistent with the result obtained by Guillemot et al. (2012), who have estimated the fraction of the pulsed emissions to be about $30 \%$ of the total emissions.

X-ray tails around pulsars have been interpreted as bow shocks generated by the supersonic motion of pulsars through space, with the wind trailing behind as its particles are swept back by the pulsar's interaction with the interstellar gas it encounters. Studying the diffuse X-ray emission may help to better understand their ambient environment and the interaction between the pulsar and the ISM. Until now, only two MSPs, PSR B1957+20 (Stappers et al. 2003) and PSR J2124-3358 (Hui \& Becker 2006), have been found to be associated with extended X-ray emission. It is worth revisiting PSR B1957+20 with a deep Chandra observation. A $\sim 25^{\prime \prime} \mathrm{X}$-ray tail extending from PSR B1957+20 with a position angle opposite to the pulsar's proper-motion direction is clearly resolved in the Chandra ACIS image. The non-thermal nature and a marginal softening of the spectrum of the tail in X-rays, as a function of the distance from the pulsar, support the scenario that particles injected at the termination shock are dominated by synchrotron cooling.
Cheng et al. (2006) suggest that the observed length $(l)$ of the X-ray tail can be interpreted as the distance traversed by the pulsar within the electron synchrotron cooling timescale $\left(t_{c}\right)$, i.e., $l \sim v_{p} t_{c}$, where $v_{p}$ is the proper-motion velocity of the pulsar. The cooling time in the X-ray band is $\sim 10^{8} B_{\mathrm{mG}}^{-3 / 2}\left(h v_{\mathrm{X}} / \mathrm{keV}\right)^{-1 / 2} \mathrm{~s}$, where $B_{\mathrm{mG}}$ is the inferred magnetic field strength in the emitting region (Cheng et al. 2006). Adopting the inferred tail length of $\sim 9.7 \times 10^{17} \mathrm{~cm}$ at a distance of $2.5 \mathrm{kpc}$ (Cordes \& Lazio 2002) and a proper-motion velocity of $220 \mathrm{~km} \mathrm{~s}^{-1}$ (Arzoumanian et al. 1994), the synchrotron cooling timescale is estimated to be $\sim 4.9 \times 10^{4} \mathrm{yr}$. This yields a magnetic field of $B \sim 17.7 \mu \mathrm{G}$ in the shock region. Considering a magnetic field strength of $\sim 2-6 \mu \mathrm{G}$ in the ISM (see Beck et al. 2003 and references therein), we found that the magnetic field in the termination shock might be compressed by a factor of $\sim 3$, which is consistent with the estimated value reported by Kennel \& Coroniti (1984).

This project is supported by the National Science Council of the Republic of China (Taiwan) through grant NSC100-2628-M007-002-MY3 and NSC100-2923-M-007-001-MY3. A.K.H.K. gratefully acknowledges support from a Kenda Foundation Golden Jade Fellowship. C.Y.H. is supported by the National Research Foundation of Korea through grant 2011-0023383. K.S.C. is supported by the GRF Grants of the Government of the Hong Kong SAR under HKU 7009/11P. L.C.C.L. is supported by the National Science Council of the Republic of China (Taiwan) through grant NSC101-2112-M-039-001-MY3.

\section{REFERENCES}

Alpar, M. A., Cheng, A. F., Ruderman, M. A., \& Shaham, J. 1982, Nature, 300,728

Antokhin, I. I., Owocki, S. P., \& Brown, J. C. 2004, ApJ, 611, 434

Archibald, A. M., Kaspi, V. M., Bogdanov, S., et al. 2010, ApJ, 722, 88

Archibald, A. M., Stairs, I. H., Ransom, S. M., et al. 2009, Science, 324, 1411

Arzoumanian, Z., Fruchter, A. S., \& Taylor, J. H. 1994, ApJ, 426, L85

Beck, R., Shukurov, A., Sokoloff, D., \& Wielebinski, R. 2003, A\&A, 411, 99

Bogdanov, S., Archibald, A. M., Hessels, J. W. T., et al. 2011, ApJ, 742, 97

Bogdanov, S., Grindlay, J. E., \& van den Berg, M. 2005, ApJ, 630, 1029

Canto, J., Raga, A. C., \& Wilkin, F. P. 1996, ApJ, 469, 729

Cheng, K. S., Taam, R. E., \& Wang, W. 2006, ApJ, 641, 427

Cordes, J. M., \& Lazio, T. J. W. 2002, arXiv:astro-ph/0207156

D’Amico, N., Possenti, A., Manchester, R. N., et al. 2001, ApJ, 561, L89

Eichler, D., \& Usov, V. 1993, ApJ, 402, 271

Fruchter, A. S., Berman, G., Bower, G., et al. 1990, ApJ, 351, 642

Fruchter, A. S., Gunn, J. E., Lauer, T. R., \& Dressler, A. 1988a, Nature, 334,686

Fruchter, A. S., Stinebring, D. R., \& Taylor, J. H. 1988b, Nature, 333, 237

Guillemot, L., Johnson, T. J., Venter, C., et al. 2012, ApJ, 744, 33

Huang, H. H., \& Becker, W. 2007, A\&A, 463, L5

Huang, R. H. H., \& Becker, W. 2010, A\&A, 510, A67

Hui, C. Y., \& Becker, W. 2006, A\&A, 448, L13

Hui, C. Y., Huang, R. H. H., Trepl, L., et al. 2012, ApJ, 747, 74

Johnson, S. P., \& Wang, Q. D. 2010, MNRAS, 408, 1216

Kennel, C. F., \& Coroniti, F. V. 1984, ApJ, 283, 694

Kulkarni, S. R., \& Hester, J. J. 1988, Nature, 335, 801

Kulkarni, S. R., Phinney, E. S., Evans, C. R., \& Hasinger, G. 1992, Nature, 359,300

Radhakrishnan, V., \& Srinivasan, G. 1982, Curr. Sci., 51, 1096

Rasio, F. A., Shapiro, S. L., \& Teukolsky, S. A. 1989, ApJ, 342, 934

Reynolds, M. T., Callanan, P. J., Fruchter, A. S., et al. 2007, MNRAS, 379, 1117

Stappers, B. W., Bailes, M., Lyne, A. G., et al. 1996, ApJ, 465, L119

Stappers, B. W., Gaensler, B. M., Kaspi, V. M., van der Klis, M., \& Lewin, W. H. G. 2003, Science, 299, 1372

Takata, J., Cheng, K. S., \& Taam, R. E. 2012, ApJ, 745, 100

Tam, P. H. T., Hui, C. Y., Huang, R. H. H., et al. 2010, ApJ, 724, L207

van Paradijs, J., Allington-Smith, J., Callanan, P., et al. 1988, Nature, 334, 684 Zavlin, V. E. 2007, Ap\&SS, 308, 297 